\title{
Traceable Air Baggage Handling System Based on RFID Tags in the Airport
}

\section{Ting Zhang ${ }^{1}$, Yuanxin Ouyang ${ }^{2}$, Yang $\mathrm{He}^{3}$}

School of Computer Science and Engineering, Beijing University of Aeronautics and Astronautics, China ${ }^{1}$ zhangting@cse.buaa.edu.cn, ${ }^{2}$ oyyx@buaa.edu.cn, ${ }^{3}$ heyang@cse.buaa.edu.cn

Received 1 August 2007; received in revised form 7 January 2008; accepted 18 February 2008

\section{Abstract}

The RFID is not only a feasible, novel, and cost-effective candidate for daily object identification but it is also considered as a significant tool to provide traceable visibility along different stages of the aviation supply chain. In the air baggage handing application, the RFID tags are used to enhance the ability for baggage tracking, dispatching and conveyance so as to improve the management efficiency and the users' satisfaction. We surveyed current related work and introduce the IATA RP1740c protocol used for the standard to recognize the baggage tags. One distributed aviation baggage traceable application is designed based on the RFID networks. We describe the RFID-based baggage tracking experiment in the BCIA (Beijing Capital International Airport). In this experiment the tags are sealed in the printed baggage label and the RFID readers are fixed in the certain interested positions of the BHS in the Terminal 2. We measure the accurate recognition rate and monitor the baggage's real-time situation on the monitor's screen. Through the analysis of the measured results within two months we emphasize the advantage of the adoption of RFID tags in this high noisy BHS environment. The economical benefits achieved by the extensive deployment of RFID in the baggage handing system are also outlined.

Key words: RFID, BHS, Tracking, Airport 


\section{Introduction}

In the last few years, Radio Frequency Identification (RFID) has become one of the most promising research areas and has attracted increasing attentions. The fundamental RFID system is composed of three components: Transponder/Tag, Reader and Backend Application. The tag consists of a microchip that stores data and antenna. It is assigned a unique serial number to identify the object item and can also store information such as price, time, date, manufacture, and product composition. Some tags are even featured with certain computing capability to realize simple data cryptograph and access control. The reader consists of the RF module, control unit, and coupling element to interrogate tags via RF communication. It also has a secondary interface to communicate with backend systems for the transmission of the information stored in tags. The backend applications not only aggregate, filter, and calculate the data gathered by readers but also process the dynamic product data (e.g. location, history and current analysis). The RFID virtually creates a remote database which travels with the items [22] by making use of RF communication to exchange data between tags and backend applications.

RFID is widely deployed in many business domains. It especially provides obvious benefits to the supply chain owing to its low cost as well as flexibility. The demand for RFID Systems is ever increasing. P. Harrop [9] indicates that the RFID industry will increase from US \$2.8 billion in 2006 to \$26 billion in 2016. Compared with other traditional automatic identify technology, RFID has advantages such as contact-less, multi-object recognition, non-line-of-sight, long distance, large store memory, programmability and penetrability. The most significant meaning embedded in the RFID technology is the fact that all the existing physical objects can enter the virtual world built by the networked RFID system. The tag can be recognized as a unique entity in the internet of physical things. The RFID network brought forward by EPCglobal or UIDCenter facilitates asset tracking and warehousing management and the essential business information can be captured or processed at any points in the transaction workflow. This information sharing in the supply chain can improve economic performance dramatically by reducing uncertainty [25]. The whole chain becomes transparent for the manufacturers, distributors and retailers to continuously monitor each other's work performance in real time. The tracking information about the inventory, for instance, the change of stock or the shipping conditions, affords references for decision-making. Moreover, global unique numbering schemes of RFID tags, for example, the Electronic Product Code (EPC) [6] enable the product's identification on the item level. For example, if the 96-bit EPC C1 G2 UHF tag is used, $2^{36}$ individual objects with the same 24-bit 'Object Class' number can be distinguished. This item-level product information capture causes more accurate lifetime traceability. It reduces the human labor costs in product delivery or replenishment and prevents product theft or counterfeiting. Y.M. Lee et al. [16] briefly demonstrate the remarkable effects provided by RFID in three factors named 'inventory accuracy', 'shelf replenishment policy', and 'inventory visibility through out the entire supply chain'.

The RFID technology is being driven by promising efficiencies not only in supply chain management and manufacturing logistics but also in the aviation logistics industry. As we all know, RFID allows the wireless storage and automatic retrieval of data. It provides a significant improvement in efficiency and security over the barcode that is used in most airports around the world. IATA (International Air Transport Association) has developed the plan for the adoption of RFID in aviation industry (e.g. baggage tag, baggage track, boarding pass, and employee pass). When RFID technology is adopted, baggage errors including mislaid baggage (occurring to approximately 40 million bags each year), lost baggage (about 50 thousand a year) and damaged baggage in aviation industry will decrease by at least 10\% [23], [10]. Our case study in Beijing Capital International Airport (BCIA) is to use the RFID tag and develop one RFID-based system to support baggage handling and baggage tracking. It is suggested that baggage in the airport can not only be assembled as well as checked more precisely but also be traced within global world.

\section{Background}

\subsection{Standard}

There are two important standards that will play significant roles in the supply chain. One is the ISO 18000 serial standard developed by the International Standards Organization (ISO), the other is the EPC standard advanced by EPCglobal. The EPC standard covers the air interfaces, the format for the product identification data stored in the RFID tag, the middleware [31] and databases storing information about tags. The EPC Class 1 Generation 2 UHF Specification submitted by EPCglobal has been compliant with the ISO 18000-6C standard. It defines requirements for a passive-back scatter, Interrogator talk first, radio-frequency identification system operating in the $860 \mathrm{MHz}$ $960 \mathrm{MHz}$ frequency range. This frequency range overcomes the differences in the UHF radio spectrum allocation for RFID among diverse areas such as North America (from 902 to $928 \mathrm{MHz}$ ), Europe (from 865.6 to $867.6 \mathrm{MHz}$ ) and Japan (from 953 to $954 \mathrm{MHz}$ ) [19]. An interrogator (known as Reader) is responsible for receiving information from a tag by transmitting a continuous-wave RF signal to the tag. The tag responds by modulating the reflection coefficient of its antenna, thereby backscattering an information signal to the interrogator. The tag is passive and receives both information and operating energy from the RF signal [5]. The aviation baggage tag's specification is suggested to operate in the UHF frequency band because UHF works well with dry, non-metallic environments. 


\subsection{IATA}

In terms of requirements in the baggage handling, the IATA associated with the airline companies, airports and RFID venders addresses the open protocol named RP1740c [3] [11]. It defines that the air interface employed is the ISO 18000-6C standard and the application protocol uses the ISO/IEC 15961 \& 15962 which respectively delineate data compression for storage and identify commands that are available to interact with the tag. Other protocols involved in our study from IATA are also shown as Table 1 (based on [17]).

Table 1: Protocols Involved in Our Work

\begin{tabular}{|l|l|l|}
\hline \multicolumn{1}{|c|}{ Object } & \multicolumn{1}{|c|}{ IATA } & \multicolumn{1}{c|}{ Scope \& Description } \\
\hline Baggage Tag & $\begin{array}{l}\text { IATA Resolution 740 } \\
\text { (IATA License Plate) }\end{array}$ & $\begin{array}{l}\text { The License Plate Number (LPN) is a unique 10 digit number (1 } \\
\text { digit: prefix to indicate general classification of tag; 3 digit: airline } \\
\text { ticket number; 6 digit: serial number) generated by accepting check- } \\
\text { in system, which enables the identification of a single bag. The LPN } \\
\text { is visually printed on each baggage strip. }\end{array}$ \\
\hline $\begin{array}{l}\text { RFID Standard } \\
\text { for Baggage } \\
\text { Tags }\end{array}$ & $\begin{array}{l}\text { IATA Recommended } \\
\text { Practice RP1740c } \\
\begin{array}{l}\text { Baggage } \\
\text { Mervices }\end{array}\end{array}$ & $\begin{array}{l}\text { Endorses the use of ultra-high frequency tags and readers } \\
\text { (interrogators) compliant with the ISO 18000-6C protocol as a global } \\
\text { air interface standard for RFID baggage tags. }\end{array}$ \\
\hline
\end{tabular}

\subsection{Airports \& Airlines}

Nearly two billion bags are shipped each year by airlines. The sector of the RFID tags market in airline baggage is scheduled to rise from $\$ 20$ million in 2006 to $\$ 100$ million in 2016 [9]. RFID supplies a real time and accurate view of the baggage along the transportation, and enormously enhances the ability for baggage sorting, baggage matching and baggage tracking, so many airlines or airports, for example, Hong Kong International Airport, Delta Airlines, McCarran International airport, British Airways/Heathrow Airport, Singapore Airlines, and Narita International Airport, have been working on the conversion from the traditional barcode-based baggage handling to RFID [10]. There are two kinds of modes for RFID-based BHS adoption. In the first application form, cheap tags (about \$0.25 per tag cost) are used to index a unique piece of baggage, just like one plate for one car. The baggage information such as the owner, the origin and the destination can be inquired about only when the background database is connected. The superiority of this mode is that the cost of the one-off tag is low and the realization of the reader's software is easy, but the back infrastructure is complex and off-line baggage information disposal is not supported. In the other form, costly tags with more memory are used to store the baggage's detailed information and the baggage's situation can be recorded at every stage after it enters the airport. The implementation of front-end reader is more complicated, but the burden on the back-end system is mitigated and the off-line query by handheld reader or else is supported. In this mode, the cost of tag is higher so the tag may be recycled.

The split of value sales of RFID systems including tags in the air industry shows the percentage spent on baggage reaches $50 \%$ and those spent on tickets, boarding passes, conveyances, vehicles, aircraft parts and others share the remaining 50\% [9]. We can sense the current developmental tendency and the tremendous economic and social potential of RFID-based baggage handling. On the other hand, although there have been some trial or rollout activities about the RFID-based applications in the air industry, RFID technology is a relative new technology. We should investigate its effects carefully before its extensive adoption.

\section{Application}

\subsection{Design}

The Beijing Capital International Airport is the largest and most important air harbor in China [1]. In 2005, the Capital airport handled more than 40 million person-time of passengers and this number will reach 60 million in 2015. According to these statistics, the requirement of doing its endeavor to avoid baggage mishandling including delayed, lost, stolen, and not-located baggage is put on the calendar, especially, considering that the coming of 2008 Olympics will accelerate the increase of the air baggage in Beijing. As a rule, passengers are assisted directly by the airline, but baggage is handled by local handling providers or airports, which, to make matters worse, change from one stage of the baggage's journey to the next. IATA in [4] surveys that the major reasons why baggage is mislaid include barcodes on the baggage not having been read properly, airplane connection times being too short and aircraft being delayed. In this paper we test the RFID's behavior on overcoming the barcode read rate and missing messages. 
(2008 Universidad de Talca - Chile

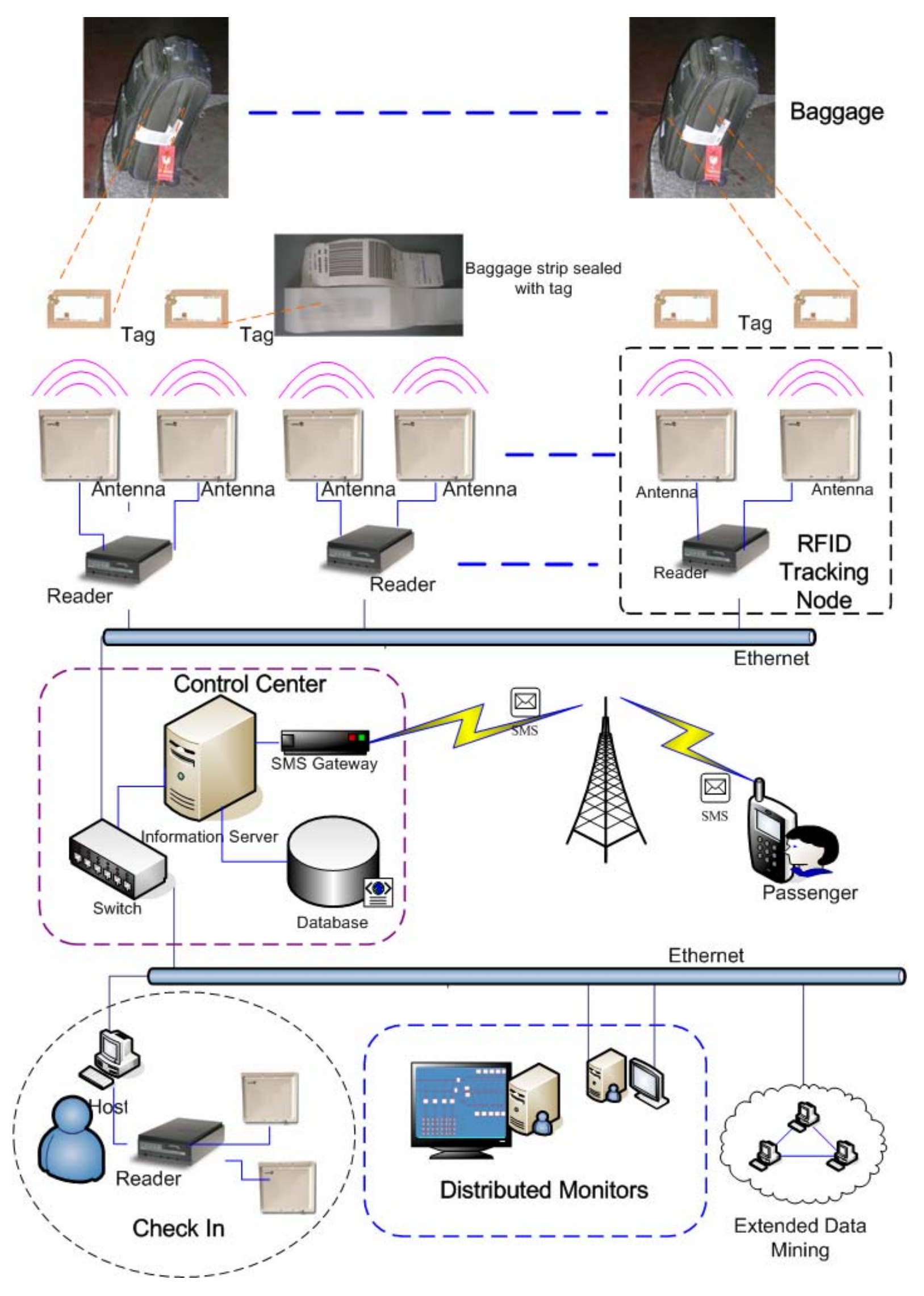

Figure 1: Topology of the RFID-Based Traceable Air Baggage Handling System

Our experimental system is shown as Figure 1. The UHF tag is sealed in the barcode-printed baggage strip and dual mode operations for baggage handling are both enabled. This approach makes RFID complement to the industry's current barcode-based automated handling systems with less efforts and investments. One RFID Tracking Node 
comprises one TCP/IP supported reader and two or three fixed antennae transmitting RF signal from different directions. Each node is installed on one pivotal position of the handling area to scan and update the information of the baggage passing by. All of the baggage strips sealed with tag and the tracking nodes compose the physical foundation tier of the system. When a departure passenger checks in, the license plate number is not only printed on the strip in human-readable barcode form but is also translated to be stored in one memory block of the RFID tag sealed in the strip. Along with the LPN, the Carrier ID, Destination, Date, Time, Passenger Name, Passenger Mobile, $\mathrm{CRC}$ and etc. are written in memory in the tag. The Control Center, including modules such as the reader manager and event manager, takes charge for the harmonization of large-scale and even heterogeneous readers. The original data inquired from RFID tracking nodes are too large so the raw data or events related to the tags must be filtered, aggregated, and cleaned to reduce the redundancies. For instance, the duplicate records owing to multiple readings of one bag at the same location are merged. After filtering and smoothing, the tag reading data in the Database can be put on varieties of compression and complex event processing. There are some tradeoffs to consider when utilizing these further operations, for example, when the system scale is within one terminal of the airport, these operations can be omitted. The Information Server mainly supplies the service interfaces and APIs to human interaction related applications by means of Web Services. These provided services emphasize on the query concerning location with baggage. The query results can be displayed on the GIS map in Distributed Monitors. Moreover, some services can be pushed to users' wireless mobile terminals, for instance, reminding the passenger that his bag has been loaded on the aircraft successfully when the right situation is detected by RFID Tracking Node. Through the meaning information abstracted from the primitive data we track all local and transfer baggage from customer check-in to placement into containers for aircraft on the GUI of distributed monitors.

\subsection{Implementation}

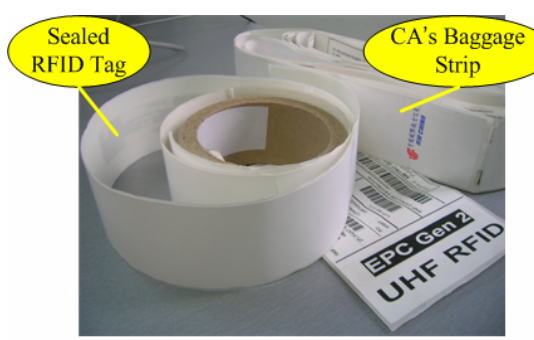

(a)

\begin{tabular}{|c|c|c|c|c|c|c|c|c|}
\hline MsgLen & Flags & Command & TagType & Address & BlockNo & DataLen & Data & CRC \\
\hline 2 (bytes) & 2 & 2 & 2 & 2 & 2 & 2 & |DataLen $\mid$ & 2 \\
\hline
\end{tabular}

Request $(\mathrm{R}-\mathrm{T})$

\begin{tabular}{|c|c|c|c|c|c|}
\hline MsgLen & RepCode & TagType & DataLen & Data & CRC \\
\hline 2 (bytes) & 2 & 2 & 2 & $\mid$ DataLen $\mid$ & 2 \\
\hline
\end{tabular}

Response $_{(\mathrm{T}>\mathrm{R})}$

(b)

Figure 2: (a) Tags for Implementation (b) Defined Protocol of Reading/Writing Command between Read and Tag

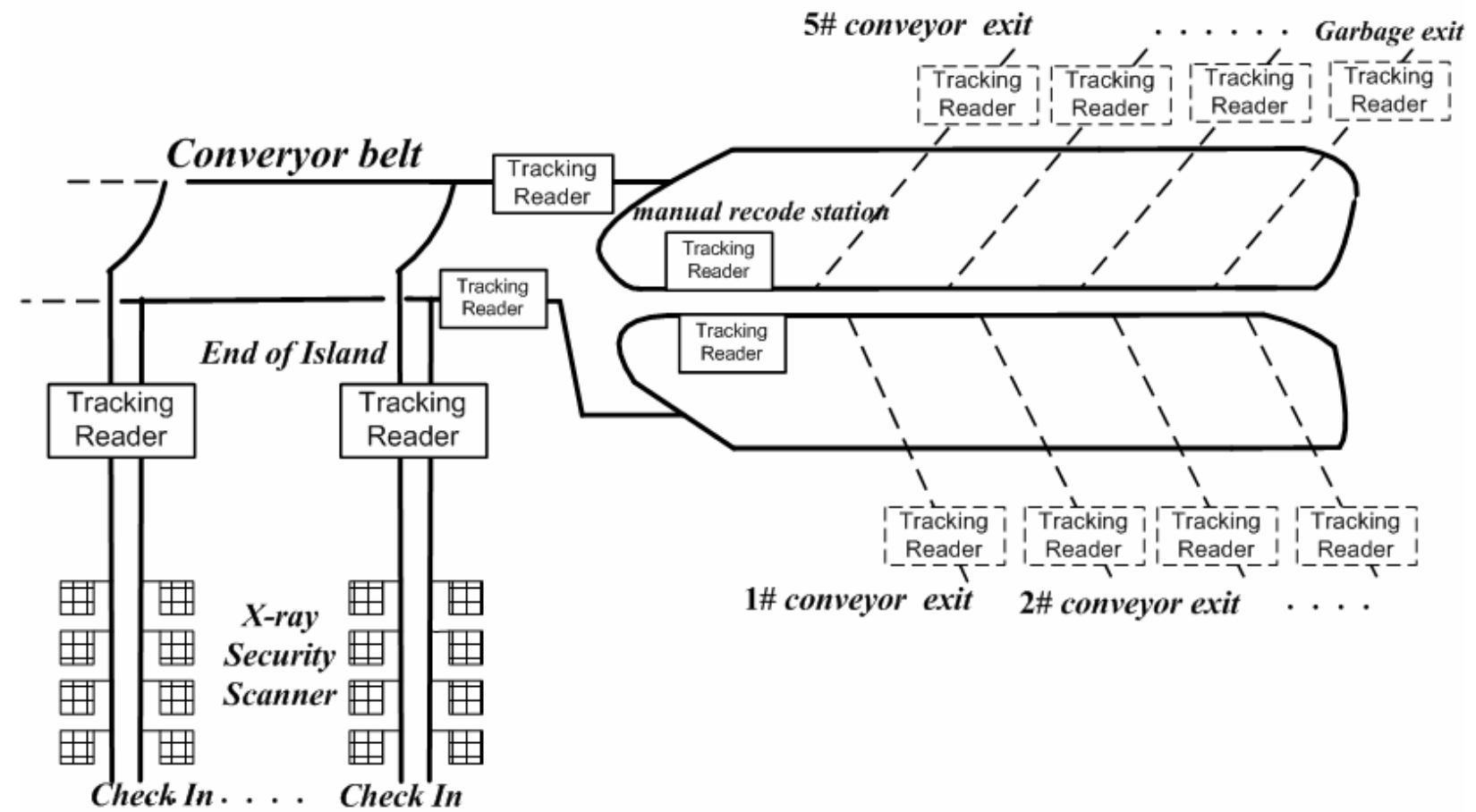

Figure 3: Layout of the System Implementation inside the BCIA Terminal 2

The application is implemented inside the BCIA Terminal 2, which is one of the busiest passenger terminals in China and contains nearly all the airlines' domestic and international flights depart from Beijing. In each baggage strip one 
RFID EPC C1 G2 UHF tag from 'UPM Raflatac' [27] is sealed (as shown in Figure 2-a). In this case, one tag matches a piece of baggage and corresponds to a passenger with his E-TKT. These Gen 2 tags are compliant with the ISO 18000-6C and more easily accessed. We deploy the readers named 'Intermec IF5' [15] (as shown in Figure 1) with three antennae in the tracking zones among the T2 BHS area, for instance, the place behind the security check area, the end of the check-in island, baggage conveyor belt, manual recode station, and sorting exit for each flight (as shown in Figure 3). All the readers are connected to the control center by the TCP/IP Ethernet. By the command packet (Figure 2-b) sent from the reader, the tag can be read at a distance by any of the antennae and a number of bags can be read simultaneously. The command request/response format depicts the protocol between the reader and the tag. Where, the 'Flags' is designate the tag query mode is select, inventory or loop; the 'TagType' describes the object tag's type such as EPC C1 Gen2, ISO 18000-C or auto-detect; the 'Address' and 'BlockNo' specifics the memory bank for the tag's reading or writing; the 'CRC' is the calculated CCITT-16 cyclic redundancy check.

We install one LED screen above each conveyor exit which is arranged for one certain scheduled flight in advance. The screen displays the amount of the sorted and sorting baggage of this flight. The distributed monitor shows the number of pieces of baggage sorted correctly and the number transferred to the error sorting conveyor exit. Considering the SMS has gained huge popularity as a medium for mobile data communication, the GPRS Web gateway supplied by 'China Mobile' is fixed in the control center. We invoke the function sendSMS (String content, String mobilePhones, String priority, String messageFlag, String moduleName, String exNumber) in ISendSMS.jsp to deliver the confirmation message indicating whether the baggage has been sorted properly before the passenger boards the airplane. The utilization of SMS will enable a real-time feedback for passengers and improve the passengers' satisfaction.

\subsection{Experimental Results}

The processing results are measured in the harsh physical environments of the baggage handling system. For one certain scheduled flight of AIR CHINA (CA) between two months (from May 16, 2006 to June 19, 2006), we calculate the read rate of the departure baggage on condition that RFID is introduced. Here, the read rate is defined as follows:

$$
\text { ReadRate }=\frac{\text { CorretHandledNumber }}{\text { TotalNumber }} * 100 \%
$$

For the experimental scheduled flight, the number of the correctly handled pieces of baggage which transit the accurate conveyor exit point automatic increase on the distributed monitors' GUl. The misread baggage is all transferred to the named 'garbage' conveyor exit where these pieces of baggage will be scanned by hand held reader and sorted manually. In this experiment, the baggage's amount does not cover all the flights of BCIA, but the experimental data in nearly two months for one flight can reflect the baggage delivery performance of RFID solution. In terms of the results shown in Figure 4, we discover that the average of varying baggage read rates achieves $96.86 \%$, which is a notable advance compared with barcode-only based system (according to the IATA, the barcode baggage tag read rates average 85\%, though other industry experts put that average at 75\%. [18] [20]). In addition, the added visibility of the baggage accurate tracking on the distributed monitors improves the worker's process control ability of the industrial field and helps to strengthen baggage security.

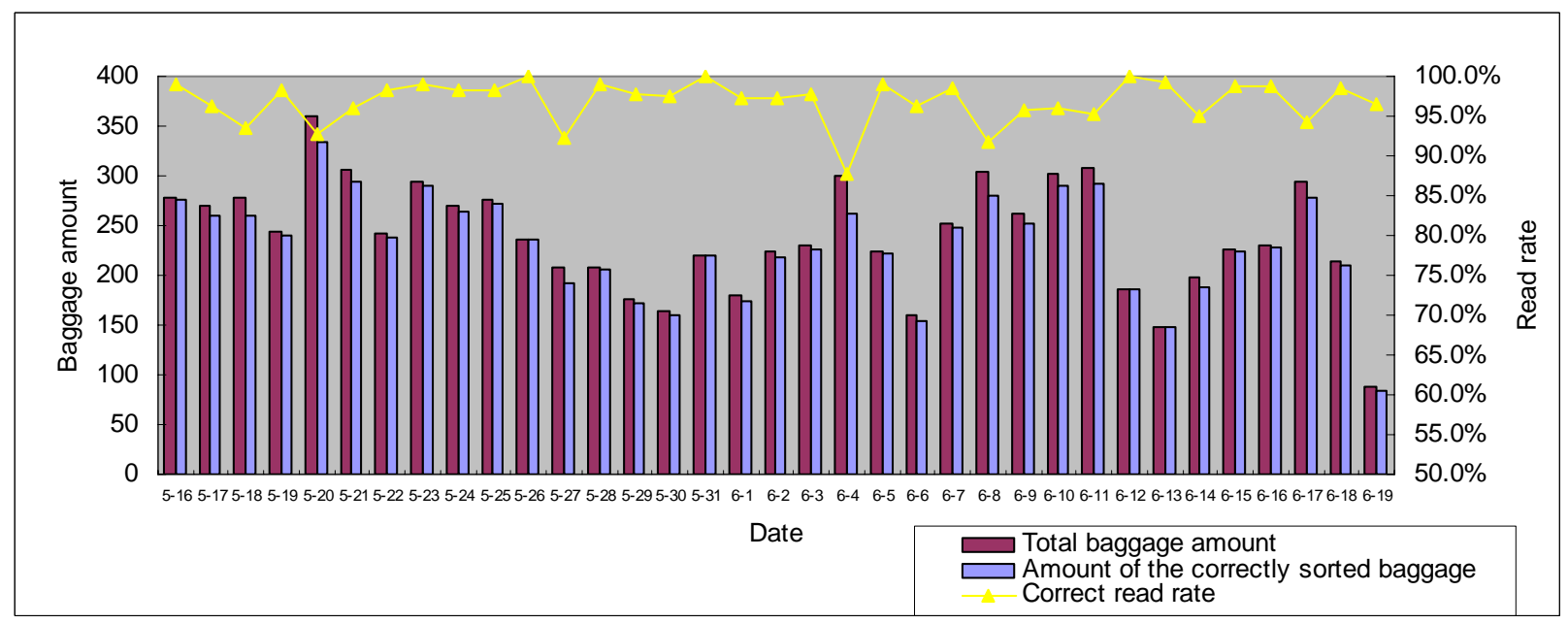

Figure 4: Read Rate of the Experimental Results for RFID-Based BHS 


\section{Benefits}

\subsection{Cost Savings}

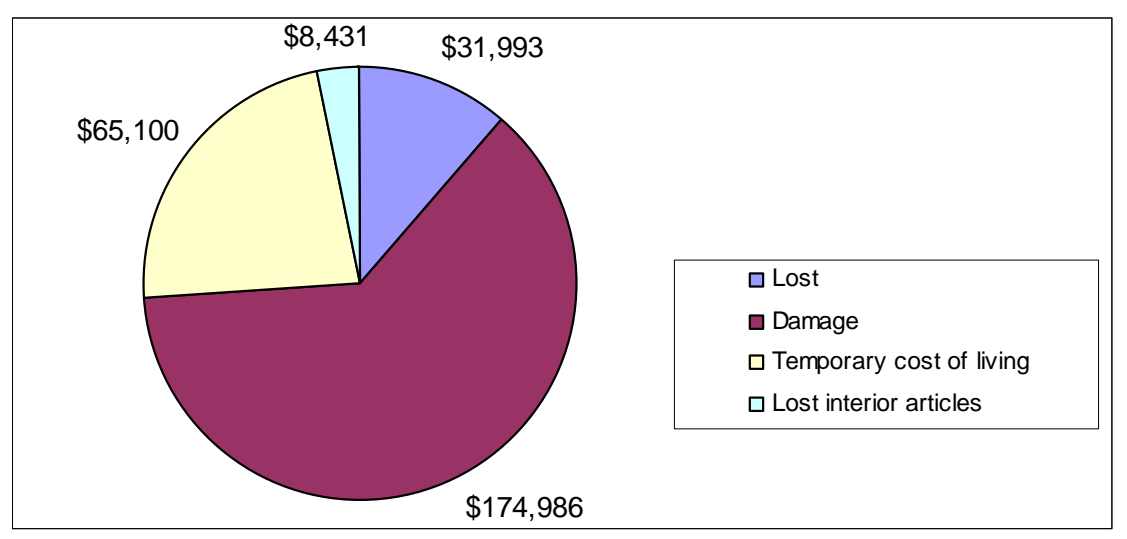

Figure 5: Compensation Cost of CA in One Year

According to the statistics from the Air China between March 19, 2005 and April 18, 2006, the compensation for lost baggage, baggage damage, temporary cost of living and lost interior articles of baggage reaches $\$ 31,993, \$ 174,986$, $\$ 65,100$ and $\$ 8,431$ respectively owing to the baggage errors (as shown in Figure 5). The reducing of misread probability and error rate will cut down this compensation cost for the airlines. In the aviation industry, out of 2 billion plus pieces of baggage handled per year, over 1\% are mishandled and each instance of baggage mishandling costs on average $\$ 90$ [12]. Upon full implementation, RFID will save the industry US\$760 million annually (based on US\$0.10 per tag cost) [6]. Furthermore, the processing cost including the labor and the maintenance cost is decreased due to the boosted productivity with a more automatic workflow brought by RFID. For example, in Hong Kong International Airport, the average cost of handling bags has gone from \$7 per bag to \$4 [15], which is a huge saving, and the overall cost savings for Hong Kong airport are estimated at more than US\$3.8 million a year [26].

\subsection{Efficiency Improvement}

Unlike reading barcodes, the reading of tags is not influenced even when they are dirty, partially folded, concealed or blocked. This characteristic, which is suitable for belt conveyors and container type sorters, effectively changes operation flow and dramatically reduces the manual processing speed and time. The staffing levels for baggage handling are also reduced because of the decrease in the effort required to sort and load bags. Owing to the simultaneous scan of multiple tags, the utilization of RFID facilitates the creation of the baggage loading list for the universal loading device (ULD) and simplifies the bag loading operation. The ULD is an important unit to store and load the passenger baggage separated by destination. When there is a RFID field in front of the ULDs, If the RFID tag on one piece of baggage whose destination is not suited passes through this field, the system distinguishes the bag from other items and audibly advises the handler whether that bag is being placed correctly or not, so manual barcode scanning and pasting are no longer required when loading baggage into these containers. Accordingly, using RFID to manifest exactly which baggage is in which container, support the matching of the baggage to passenger 'aboard aircraft' status, and locate the container which holds the passenger baggage, is valuable from the security as well as operational efficiency standpoints [2]. Furthermore, the bags transferred to error sorting conveyor or late bags are read by WiFi-enabled handheld RFID reader linked to the control center to acquire their information for delivery promptly. The lessening of the amount of handling time and errors is also important financially, since every air carrier in the world represents delay impact in terms of 'cost per delay minute [2]'.

\subsection{Potential Worthiness}

U.S. Department of Transportation [28] surveys that the baggage now ranks second on the customer complaints list, and the ranking is likely to worsen in the coming years. The problems (e.g. long baggage claim time, delayed, damaged, and lost baggage) affect customer satisfaction with potential direct loss of business. The effects of decreasing customer satisfaction also put future revenue at risk [29]. Depending on [10], the passenger claims will be reduced by 5.7 million when RFID technology is adopted. Furthermore, the RFID-base traceable system makes the passenger be more aware of the airport baggage performances and affects his airlines or airports choice in his purchase decision. RFID enables remote programming of tags and opens up the potential to write accessional data into tags. Additionally, more added-value services can be integrated into the system facilely, such as security check operation, real time location systems to track ground service equipment and people around airports (using active tags), RFID-based access control systems, and RFID for cargo. The RFID data mining on the baggage database also enables specific friendly or VIP services to be pushed in terms of the passenger's attribute, behavior pattern or 
interest, for instance, providing personalized reminding service according to the location context of passenger and his baggage.

\section{Concerns}

\subsection{Investment}

As discussed, barcodes require optical line of sight and can be blocked by many materials that are transparent to RFID. In the air baggage handling environment which is harsh and noisy, the barcode-based system has many disadvantages [8] (see Table 2), such as the influence of baggage moving, barcode wear or envelop. When considering the transition from barcode to RFID, the investment is the remaining crucial consideration in the widespread adoption. On the contrary to the barcode, the price of one RFID reader $(4,000 \$)$ is cheaper than that of one long-range barcode reader $(15,000 \$)$, but the cost for the RFID tag is more expensive. The current passive tag prices, which are dozens of times the barcode price, range from $\$ 0.15$ to $\$ 1.10$ depending upon the volume of tags produced and the complexity of tag functions [32]. The high cost of RFID tag is a major reason why the penetration of RFID for aviation logistics remains stagnant but RFID is getting cheaper due to innovation in the manufacture as well as the usage on a larger scale. In fact, there have been recent press released by tag suppliers such as Alien Technologies [21] and SmartCode [24] announcing 12.9 and 5 cents per tag respectively. IATA [13] believes that RFID will continue to decrease in cost through innovations such as silicon free RFID in a 6 - 8 years timeframe and David Vollmer [30] even forecasts that the sub 1 cent tags are 5 to 10 years out. The decrease of the tags' price drives up the RFID system installations in airport and airline domains while the increase in the volume of demand is a factor to reducing costs responsively, in respect that RFID tags for a major airline are in volumes of 10 million per year [10]. The market of civil aviation in China is going up by $7.6 \%$ annually, which is ranked second in the world, next to the U.S.A.. This rising requirement in the aviation passenger transportation ensures the return on investment in the RFID setting. As discussed, expectations of return can be broken down into two parts: The first is cost reduction (labor cost reduction, inventory cost reduction, process automation, and so on), and the second is value creation (increase in revenue, increase in customer satisfaction due to responsiveness, and so on) [32]. More positive returns on investment can be achieved by the cooperation between airports and airlines along with comprehensive RFID adoption. The airport is considered to be suitable to play the major role in the initial RFID system investment including hardware. The operating costs owing to the consumed tags should be burdened by airports associated with airline companies according to the amount of passengers.

Table 2: Comparisons of RFID with Barcode in the BHS

\begin{tabular}{|c|c|c|c|c|c|c|c|c|c|c|c|}
\hline Type & Range & $\begin{array}{l}\text { Anti- } \\
\text { collision }\end{array}$ & Memory & $\begin{array}{c}\text { Program- } \\
\text { mable }\end{array}$ & $\begin{array}{l}\text { Read- } \\
\text { ability by } \\
\text { people }\end{array}$ & $\begin{array}{l}\text { Penetr- } \\
\text { ability }\end{array}$ & $\begin{array}{l}\text { Influence of } \\
\text { dirt/damp }\end{array}$ & $\begin{array}{l}\text { Degrada- } \\
\text { tion/wear }\end{array}$ & $\begin{array}{l}\text { Reading } \\
\text { speed }\end{array}$ & $\begin{array}{l}\text { Encry- } \\
\text { ption }\end{array}$ & $\begin{array}{c}\text { Invest- } \\
\text { ment }\end{array}$ \\
\hline Barcode & $<10 \mathrm{~cm}$ & No & Small & Limited & Limited & $\begin{array}{r}\text { Total } \\
\text { failure }\end{array}$ & Very high & Limited & $\begin{array}{l}\text { Low } \\
\sim 4 \mathrm{~s}\end{array}$ & No & $\begin{array}{c}\text { Reader: } \\
\text { High } \\
\text { Barcode: } \\
\text { Low }\end{array}$ \\
\hline $\begin{array}{c}\text { RFID } \\
\text { (UHF } \\
\text { EPC C1 } \\
\text { G2) }\end{array}$ & $<10 m$ & Yes & Large & Flexible & $\begin{array}{c}\text { Impossibl } \\
\mathrm{e}\end{array}$ & Good & No influence & $\begin{array}{c}\text { No } \\
\text { influence }\end{array}$ & $\begin{array}{c}\text { Very fast } \\
<1600 \text { tags/s }\end{array}$ & Yes & $\begin{array}{c}\text { Reader: } \\
\text { Low } \\
\text { Tag: High }\end{array}$ \\
\hline
\end{tabular}

\subsection{Infrastructure}

The building of a UHF RFID infrastructure across the entire aviation industry chain is figured as indispensable to obtain more real effectiveness. IATA analysis in [14] presents that the top 80 airports need to be equipped with RFID to get global benefits, as this can cover $80 \%$ of all baggage mishandling. In respect that the baggage travels around the world, the infrastructure should be established to collect and track the real-time data flow of tag information at every stage in the chain. The necessary information could reside in one of many different back-end information servers owned by a given airport, so the information server of every stage needs to be shared to enable a traceability solution aligned with processes that capture and store data to access any piece of baggage around the world. In order to meet this large-scale tracking need, the resolution such as ONS (Object Naming Service) [7] which uses the Internet's existing DNS (Domain Name System) for looking up or resolving information about an EPC related services should be introduced. The central and large airports are the leaders to introduce RFID into existing barcodebased BHS and the medium or small airports accomplish the transition to RFID-based BHS gradually. Therefore, barcodes will coexist with RFID for a period of time. When the global RFID infrastructure is established, all of the participators, including airports and airlines, will 'see' any piece of baggage along its travel at any time and obtain the supreme return. Also, the passenger can expediently access his baggage's information through the Internet connection. 


\section{Conclusion}

The RFID system supplies a real-time view of the item-level baggage information and provides a stronger automated solution in BHS which more value-added services can be integrated in. We present a practical RFID system adopted in the $\mathrm{BCIA}$ airport Terminal 2 for baggage trace and detail the design, implementation and performance. The results analysis indicates that RFID is compatible with airports' circumstances and available to lessen operation cost, improve operational efficiency and maintain high passenger satisfaction level. Based on the experiment in the Beijing airport, we conclude that:

- In the air baggage handling scenarios, the RFID is superior to barcodes with its advantages such as long range, anti-jamming, item-level identifier, multi-recognition. In our experiment, the RFID-based average read rate achieved $96.86 \%$ while the barcode read rates in the range of $75 \%$ \%5\%. The transition to RFID will reduce labor cost and create potential value. As a result, replacing barcode by RFID is an inevitable trend.

- $\quad$ As for RFID-based BHS, the operating cost brought by the tags will be much more than the initial setting cost owing to the readers. We suggest that the airport should be responsible for the investment to set up the system, and the investment to operate the system be shared proportionally by airports and airlines.

- $\quad$ The maximal return on investment will not be achieved until the global RFID-based network infrastructure for baggage handling has been constructed. The medium or small size airports can implement the transition to RFID gradually after the top large and crucial airports have brought the RFID-based BHS into effect. Therefore, the barcode-based and the RFID-based system will coexist for a time.

We recommend that more impacts and competitive advantages are archived in aviation industry by applying RFID in mass scenarios. Moreover, the privacy protection along the network across the BHS around the world will be emphasized on in our future work.

\section{References}

[1] Beijing Capital International Airport Co. Ltd.. Company Introduction. [Online]. Available: http://www.bcia.com.c n/en/about company page.html.

[2] A. Cerino, and W. P. Walsh, Research and Application of Radio Frequency Identification (RFID) Technology to Enhance Aviation Security, presented at the IEEE 2000 National Aerospace and Electronics Conference, Dayton, OH, October 10-12, 2000, pp. 127-135.

[3] P. Chartier. (2005). An Overview of ISO RFID Standards Applied to IATA Baggage Handling, Technical Report. [Online]. Available: http://www.iata.org/NR/rdonlyres/53329227-F16B-4FB8-A311-BADB1F431C12/0/ISORFID DataProtocol.pdf.

[4] R. Das. (2007, May). The Myth and Reality of Baggage Tagging. [Online]. Available: http://www.idtechex.com/p roducts/en/articles/00000534.asp.

[5] EPCglobal. (2006). EPC ${ }^{\mathrm{TM}}$ Radio-Frequency Identity Protocols Class-1 Generation-2 UHF RFID Protocol for Communications at $860 \mathrm{MHz}-960 \mathrm{MHz}$ Version 1.0.9. [Online]. Available: http://www.epcglobalinc.org/standar ds/uhfc1g2/uhfc1g2 10 9-standard-20050126.pdf.

[6] EPCglobal. (2006, March). EPCglobal Tag Data Standards Version 1.3. [Online]. Available: http://www.epcgloba linc.org/standards/tds/tds 1 3-standard-20060308.pdf.

[7] EPCglobal. (2006). Object Naming Service (ONS) Version 1.0 EPCglobal Ratified Specification. [Online]. Available: http://www.epcglobalinc.org/standards/ons/ons 1 0-standard-20051004.pdf.

[8] K. Finkenzeller. RFID Handbook: Fundamentals and Applications in Contactless Smart Cards and Identification (Second Edition). New York: John Wiley \& Sons, 2003.

[9] P. Harrop. (2006, August). RFID in the Air Industry and Land Transport. [Online]. Available: http://www.idteche x.com/products/en/articles/00000486.asp.

[10] International Air Transport Association. (2007). RFID Business Case for Baggage Tagging. [Online]. Available: http://www.iata.org/NR/rdonlyres/99091491-CB49-4913-BAB4-EA578CA814CC/0/RFIDforbaggagebusinesscas e21.pdf.

[11] International Air Transport Association. (2007). IATA RFID Tutorials - RFID Encoding To RP1740c. [Online]. Available: http://www.iata.org/NR/rdonlyres/FEE3B583-A9E0-4AF0-B81A-9E97A7AAD574/0/RFIDEncoding tuto rial.pdf.

[12] International Air Transport Association. (2007, August). Fact Sheet: Radio Frequency Identification (RFID) for Aviation. [Online]: Available: http://www.iata.org/pressroom/facts figures/fact sheets/RFID.htm.

[13] International Air Transport Association. (2006, January). Simplifying the Business Strategy 2006: RFID. [Online]: Available: http://www.iata.org/NR/rdonlyres/9CABFA21-BE46-426C-AA6E-9E568C1F656F/0/2006StrategyRFI D.ppt. 
[14] International Air Transport Association. (2007). RFID Transition Plan for Baggage. [Online]. Available: http://www.iata.org/NR/rdonlyres/081E47E4-6EF7-474F-AD64-C9C6671FDAF1/0/RFIDTransitionPlanfor bagga ge12.pdf.

[15] Intermec Technologies. (2006). Product Profile - IF5 Fixed Reader. [Online]. Available: http://epsfiles.interm ec.com/eps files/eps spec/lF5 spec web.pdf.

[16] Y. M. Lee, F. Cheng, and Y. T. Leung, Exploring the Impact of RFID on Supply Chain Dynamics, Proceedings of the 2004 Winter Simulation Conference, Dec. 5-8, 2004, pp. 1145-1152.

[17] m2p Consulting, Implementing RFID Tagged Baggage, The 3rd Annual Middle East Aviation IT Manager Forum, Technical Report, November, 2005.

[18] A. Moses. (2006, November). Lost in Transit no More: RFID Luggage Tracking. [Online]. Available: http://ww w.indoorlbs.com/id151.html.

[19] P. Nemeth, L. Toth, and T. Hartvanyi, Adopting RFID in Supply Chains, presented at 2006 IEEE International Conference on Mechatronics, July 2006, pp. 263-266.

[20] M. C. O'Connor. (2005, November). IATA Approves UHF for Bag Tags. [Online]. Available: http://www.rfi djournal.com/article/articleview/1994/.

[21] M. C. O'Connor. (2005, September). Alien Drops Tag Price to 12.9 Cents. [Online]: Available: http://www.rfidjo urnal.com/article/articleview/1870/1/1.

[22] E. C. Ozelkan, Y. Sireli, M. P. Munoz, and S. Mahadevan, A Decision Model to Analyze Costs and Benefits of RFID for Superior Supply Chain Performance, presented at Technology Management for the Global Future, 2006. PICMET 2006, Istanbul, Turkey, July 2006, pp. 610-617.

[23] L. Riley. (2005, November). IATA Introduces RFID Standard for Baggage Tags Annual Industry Savings Projected at US\$760 Million. [Online]. Available: http://www.iata.org/pressroom/briefings/2005-11-18-01.

[24] M. Roberti. (2006, May). SmartCode Offers 5-Cent EPC Tags. [Online]: Available: http://www.rfidjournal.com/ar ticle/articleview/2296/1/1.

[25] F. Sahin, J. Robinson, and E. Powell, Information Sharing and Coordination in Make-to-Order Supply Chains, Journal of Operations Management, vol. 23, no. 6, pp. 579-598, 2005.

[26] SITA. (2007, June). The Arrival of RFID. [Online]: Available: http://www.sita.aero/News Centre/Publications/S olutions at SITA Q2 2007/Features/The arrival of RFID.htm.

[27] UPM Raflatac. UHF Products. [Online]. Available: http://www.upmraflatac.com/asia/eng/RFIDProducts/UH FProducts.

[28] U.S. Department of Transportation. (2006, October). Air Travel Consumer Report. [Online]. Available: http://airco nsumer.ost.dot.gov/reports/atcr06.htm.

[29] C. Vincent, H. Deijkers, J. Strik, V. Atmadja, D. Polizzi and M. S. Boyce, Beyond the Carousel-Better Baggage Handling Through Enhanced Collaboration Among Airlines and Airports, IBM Institute for Business Value, NY, USA, Technical Report, 2007.

[30] D. Vollmer, RFID: From Compliance to Competitive Advantage, Sr. Logistics Consultant RedPrairie Corporation, USA, Technical Report, 2004.

[31] R. Weinstein, RFID: A Technical Overview and Its Application to the Enterprise, IT Professional, vol. 7, no. 3, pp. 27-33, 2005.

[32] N.C. Wu, M.A. Nystrom, T.R. Lin and H.C. Yu, Challenges to Global RFID Adoption, presented at Technology Management for the Global Future, 2006. PICMET 2006, Istanbul, Turkey, July 2006, pp. 618-623. 